\title{
Baselines for Suppressed Demand: CDM Projects Contribution to Poverty Alleviation
}

\author{
Harald Winkler \\ Energy and Development Research Centre, University of Cape Town
}

\section{Steve Thorne}

SouthSouthNorth Project, Cape Town

\begin{abstract}
Projects implemented under the Clean Development Mechanism (CDM) need to establish a baseline. The baselines is a projection of greenhouse gas emissions that would have occurred without the project. Establishing baselines that allow for sustainable development through CDM projects is a key challenge, especially in poor communities. The CDM rules explicitly allow for baselines that account for emissions "above current levels due to specific circumstances of host parties". This provision lends support to crediting of growth in demand for energy services where it is currently suppressed as a result of poverty and/or lack of infrastructure or suppressed demand. The question is whether the existing level of consumption is the baseline or the future expected level of consumption including "development" advances in provision of energy services and as a result of poverty alleviation is the baseline. Or should development be allowed to get dirty before it qualifies to become clean? The paper presents a baseline methodology that provides opportunities for suppressed demand to be predicted and counted.
\end{abstract}

JEL Q25, Q40

\section{INTRODUCTION}

The United Nations Framework Convention on Climate Change (UNFCCC) aims to reduce emissions of greenhouse gases (GHGs) in order to "prevent dangerous anthropogenic interference with the climate system' and promote sustainable development (UNFCCC, 1992). The Kyoto Protocol, which was adopted in 1997 and appears likely to be ratified by 2002 despite the US withdrawing, aims to provide means to achieve this objective (UNFCCC, 1997).

The Clean Development Mechanism (CDM) is one of three 'flexibility mechanisms' in the Protocol, the other two being Joint Implementation (JI) and Emisșions Trading (ET). These mechanisms allow flexibility for Annex I 
Parties (industrialised countries) to achieve reductions by extra-territorial as well as domestic activities. The underlying concept is that trade and transfer of credits will allow emissions reductions at least cost. Since the atmosphere is a global, well-mixed system, it does not matter where greenhouse gas emissions are reduced.

The CDM is a project-based mechanism that allows Annex I Parties to meet part of their emissions reductions targets by investing in developing countries. CDM projects must also meet the sustainable development objectives of the developing country. Further criteria are that Parties must participate voluntarily, that emissions reductions are 'real, measurable and long-term', and that they are additional to those that would have occurred anyway. The last requirement makes it essential to define an accurate baseline.

The crediting of increases in demand, where it has previously been suppressed, has a basis in the rules of the CDM negotiated in the UNFCCC process. Paragraph 46 of the Marrakech Accord states that: "The baseline may include a scenario where future anthropogenic emissions by sources are projected to rise above current levels, due to the specific circumstances of the host Party" (UNFCCC, 2001: 37, para. 46).

This paper argues that suppressed demand is a specific circumstance that justifies baseline scenarios in which future emissions increase. The paper introduces the concepts of baselines and suppressed demand, provides examples of baselines for CDM projects which would avoid emissions, and suggests lessons for a generic methodology for suppressed demand baselines.

\section{WHAT ARE BASELINES AND THEIR USES?}

There is a growing literature on baselines for project-based mechanisms (Lazarus et al., 1999; Bernow et al., 2000; Lazarus et al., 2000; Meyers, 2000; OECD \& IEA, 2000; Winkler et al., 2000; Lazarus et al., 2001). Put simply, baselines seek to predict what would have happened without the CDM project. If emissions from the project are lower than in the business-as-usual (BAU) case, real emission reductions will be achieved.

Baselines may be used for two purposes, (i) to determine whether the project is additional to what would have happened anyway, and (ii) to calculate the credits to be transferred between host and investor entities. The first purpose - testing additionality - occurs before the CDM project is implemented, while credits must of course be calculated after project implementation. 
Article $12(5)(c)$ of the Kyoto Protocol requires that emissions reductions be "additional to any that would occur in the absence of the certified project activity" (UNFCCC, 1997). If a project would have happened anyway, it should not be a CDM project and receive investment through that mechanism . Baselines suggest themselves as means of testing additionality (UNFCCC, 1997; Baumert, 1999; Meyers, 1999). Once a project has qualified for the CDM and been implemented, the emissions reductions need to be calculated. To do so, the difference between the baseline agreed at the start of the project and the project performance needs to be calculated. We focus here on the latter use of baselines, to calculate Certified Emissions Reductions (CERs) under CDM projects.

Like any projection, baselines depend on assumptions about the future. Key assumptions include the level of economic growth, energy supply and demand. Baselines are counterfactual (Lazarus et al., 2001), in the sense that, due to climate change policy, the baseline will never occur. A judgement is made by the persons calculating the baseline relating to the reference case - for example, if the CDM project had not installed compact fluorescent lightbulbs (CFLs), incandescents would have been used; if the CDM had not contributed to a wind power park, gas-fired electricity generation would have produced the power instead.

In calculating CERs, it is usually assumed that the same level of activity applies to both the baseline scenario and the CDM project. For example, the same number of MWh that would have been generated from a coal-fired power station are replaced by a CDM project implementing a cleaner energy technology. In situations of suppressed demand, this constraint may need to be relaxed. Before considering calculations of baselines, however, we first explain the importance of suppressed demand.

WHY IS SUPPRESSED DEMAND IMPORTANT?

In poor countries and communities, households demand less services because they cannot afford to buy more. Demand is suppressed or remains suppressed ${ }^{1}$ due to a budget constraint or lack of infrastructure. Clearly this means that sustainable development is not being achieved, in that basic human needs are not being satisfied.

In economic theory, households face a budget constraint. If they allocate their total budget between two goods, they can only consume more of one good by trading off the other (Nicholson, 1995: 107-9). So household cannot move to higher levels of utility, due to the income constraint. The suppressed demand 
situation would be where the budget constraint lies below any utility level considered to meet basic needs. Introducing energy savings means that household income increases, allowing it to move to higher levels of service (a higher utility curve).

Suppressed demand also has implications for baselines and the calculation of CERs, through the 'take-back' or 'rebound' $\operatorname{effect}^{2}$ (Greening et al., 2000). Part of the emission reduction in technical efficiency is taken back by a change in consumer behaviour. Roy (2000: 433) describes the effect as the way in which "... the benefits from savings in energy demand arising out of the technical efficiency improvement, and hence reduction in GHG emissions, are going to be taken back through increase in demand". The calculated energy savings (and hence emission reductions) are greater than the actual savings. The degree to which this happens can be defined as (Roy, 2000: 435):

\section{Rebound effect $(\%)=100 *$ (Calculated savings - Actual Savings) $/$ Calculated savings}

Energy efficiency projects are perhaps the clearest example of the effect. Consider an energy efficiency project which reduces the amount of $\mathrm{CO}_{2}$ per unit of output. Higher efficiency reduces the emissions intensity $\left(\mathrm{kgCO}_{2}\right.$ /unit output). However, the efficiency gains also results in energy savings. These savings may be invested in additional activity - the take-back effect. The additional activity can occur either extending operating hours or increasing capacity. If demand is unsatisfied or suppressed, it is very likely there will be significant take-back - to meet the unsatisfied demand.

In calculating CERs, the baseline methodology must consider how to deal with the increased level of activity represented by the take-back effect. Compared to analysis of the CDM project without the take-back effect, the annual emissions will increase.

In the extreme case, where a service (e.g. bus transport) was previously completely unavailable, the introduction of a cleaner technology may still result in an increase the absolute levels of GHG emissions. However, compared to a BAU development path (without cleaner technologies), the technology introduced through the CDM may be cleaner. In the SA energy sector, for example, the introduction of gas-fired power stations may increase total emissions, but would produce lower emissions than new coal-fired plants.

We argue that credit should be given to development paths that 'leap-frog' to cleaner technologies, without first getting dirty. The following sections outline how this might be achieved in calculating CDM project baselines. 


\section{CALCULATING CERs}

While different methodologies exist for calculating baselines (Michaelowa \& Dutschke, 1999; Meyers, 2000; OECD \& IEA, 2000; Lazarus et al., 2001; Sathaye, 2001), some generic steps can be identified. For the purpose of calculating CERs, these steps may be performed on a periodic (perhaps annual) basis:

1. Define the reference scenario (e.g. the set of power plants to compare against, the form of transport that would otherwise have been used, the lights installed, etc.);

2. Define a set within that scenario - comparing against the whole sector, recently-built plants, only best available technologies;

3. Calculate emissions intensity of baseline scenario and $\mathrm{CDM}$ project $\left(\mathrm{kg} \mathrm{CO}_{2} /\right.$ unit output).

4. Reduced emissions intensity $=$ Baseline emissions intensity - CDM project emissions intensity; and

5. Calculate CERs by multiplying by activity level (CERs = activity level * reduced emissions intensity. In units: CERs $(\mathrm{kg} \mathrm{CO} 2)=$ activity level (unit output) * reduced emissions intensity $(\mathrm{kgCO} 2 /$ unit output.

This brief outline of steps makes clear that the activity level is a key factor in determining the numbers of credits eventually transferred. How this plays itself out in a CDM project in a situation with suppressed demand is best illustrated by means of examples.

\section{EXAMPLES OF DEMAND BASELINES}

\subsection{Take-back effect: Efficient Lighting example}

A practical example of an efficient lighting project demonstrates the issues around the take-back effect and suppressed demand. CDM projects may replace incandescent lamps with compact fluorescent lightbulbs (CFLs) of a much lower wattage. Such projects have been initiated in South Africa even prior to the CDM, with the costs of the CFLs being paid by the programme. Eskom has launched an efficient lighting initiative aiming to install some 18 million CFLs (Eskom, 2000), and economic analyses have been conducted (Clark, 1997). For the purposes of this article, we focus on a single household using only electricity for lighting.

Assume that before the CDM project, the household uses two $100 \mathrm{~W}$ incandescent bulbs. Being a low-income household, they cannot afford to nun 
more bulbs. Assume that the CDM project replaces the incandescents with two CFLs of $25 \mathrm{~W}$ each. Assuming a 75 per cent increase in efficiency from incandescents to CFLs, the household receives the same amount of light with less energy consumed. Service levels (the amount of light) remain the same in this initial situation.

Initial situation, before the CDM: $2 \times 100 \mathrm{~W} \mathrm{x} 4 \mathrm{hr} / \mathrm{d} \times 30 \mathrm{~d} / \mathrm{mth}=24 \mathrm{kWh} /$ month.

After the CDM (without take back): $2 \times 25 \mathrm{~W} \times 4 \mathrm{hr} / \mathrm{d} \times 30 \mathrm{~d} / \mathrm{mth}=6 \mathrm{kWh} /$ month.

The total capacity used for lighting was decreased from $200 \mathrm{w}$ to $50 \mathrm{~W}$, and hence the monthly energy use is expected to decrease from $24 \mathrm{kWh}$ to $6 \mathrm{kWh}$. Reduced energy consumption means that the household saves on expenditure for lighting. This saving might be used to buy more lighting, which is likely in a situation where demand is suppressed by poverty. Using the energy savings to buy other services is also likely, but is considered part of the sustainable development benefits of the CDM project and is not included in the baseline calculations.

In response to this situation, the household may increase its consumption either by increasing the number of operating hours of the bulbs, or by installing more lights. For calculation purposes, the effect on the consumption in $\mathrm{kWh} /$ month of installing twice the number of bulbs or running existing bulbs two times as long is the same. In practice, there are significant differences, given the high initial costs of $\mathrm{CFL}^{3}$. We therefore assume that the increased service levels are reflected in a doubling of operating hours.

After the CDM (with take back/increased service): $2 \times 25 \mathrm{~W} \times 8 \mathrm{hr} / \mathrm{d} \times 30$ $\mathrm{d} / \mathrm{mth}=12 \cdot \mathrm{kWh} / \mathrm{mth}$.

The development benefits of the project under this scenario are two-fold. Firstly, the level of lighting has doubled from the baseline scenario. Secondly, the household still saves money on energy for lighting, paying only half the number of $\mathrm{kWh}$ per month.

Accounting for suppressed demand requires that we consider what would have happened without the CDM project. We assume that BAU economic development will lead to increased levels of service, as economic development increases incomes and better infrastructure is gradually established. Over time, doubling service levels with the old technology would have resulted in:

BAU growth: $2 \times 100 \mathrm{~W} \times 8 \mathrm{hr} / \mathrm{d} \times 30 \mathrm{~d} / \mathrm{mth}=48 \mathrm{kWh} / \mathrm{mth}$. 
The various levels of energy for lighting correspond to emissions, since we are only considering electricity for lighting. Since the lighting comes from the same grid, less energy used results in a directly proportional reduction of GHG emissions. Situations were multiple fuels are used for lighting and replaced by electricity require a separate analysis.

Three different situations can be considered with the help of rough graphic representations. The effects on consumption and service levels are shown separately in Figures 1 and 2.

A business-as-usual (BAU) path of development uses the old technology (incandescent lamps) and service levels grow gradually over time. Consumption of lighting services starts at a level where part of the demand remains unsatisfied or suppressed. The CDM project (without the take-back effect, the lowest, short-dashed line) lowers the consumption level through the increased efficiency of a new technology, in this case CFLs. Again, we assume that demand grows somewhat over time. Service levels are initially the same as in the BAU case, and increase at a comparable rate.

The solid line shows CDM project with the take-back effect. The new, more efficient technology is used, but service levels increase as the household uses energy savings to purchase more lighting services. The top line shows what development might have occurred if there was no constraint on demand. In determining the level of this line, judgements have to be made on what constitutes 'satisfied demand'. Energy consumption would increase to satisfy demand, say at 10 or 12 hours/day.

In Figure 1, the shaded areas A and B together show the difference before and after the project. Since energy consumption (and hence GHG emissions) grows over time in each case, the sum of the two areas are kWhs of electrical energy, which if multiplied by an emissions factor, represents the emissions reductions. Area B on its own represents the take-back effect, the amount of increased consumption of lighting, which the household pays for out of its energy savings. The increased lighting service is a sustainable development benefit of the project. 
Figure 1 Consumption of lighting services under business as usual, with CDM project, with the take-back effect and under development wlthout constraint

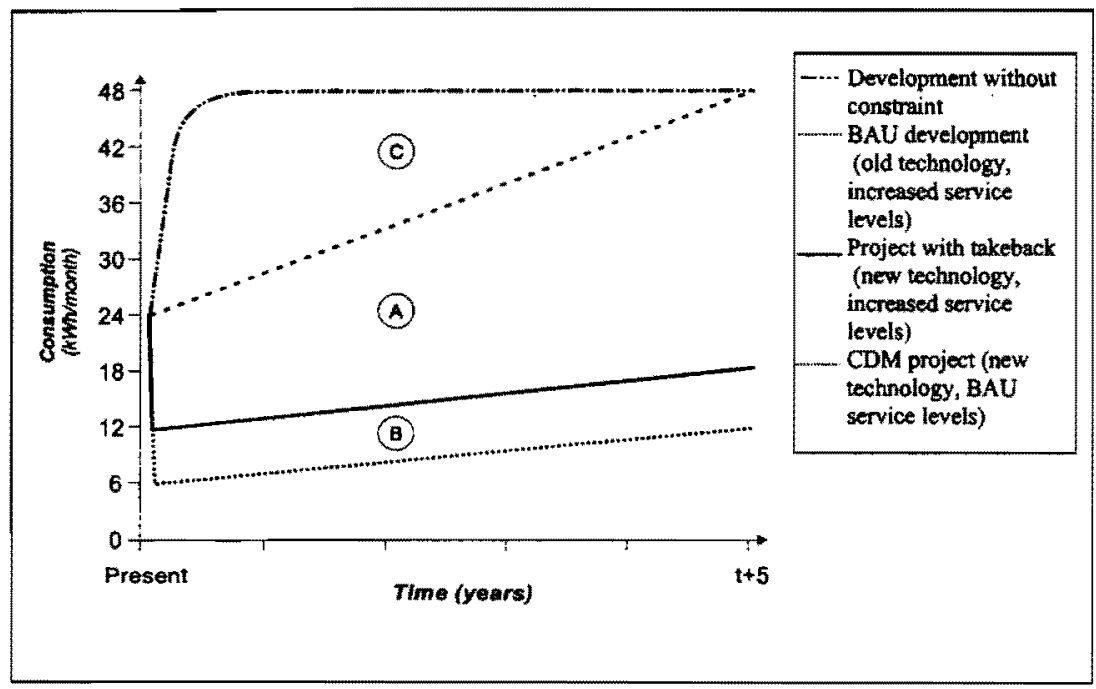

Figure 2 Service levels under business as usual, with CDM project and take-back effect

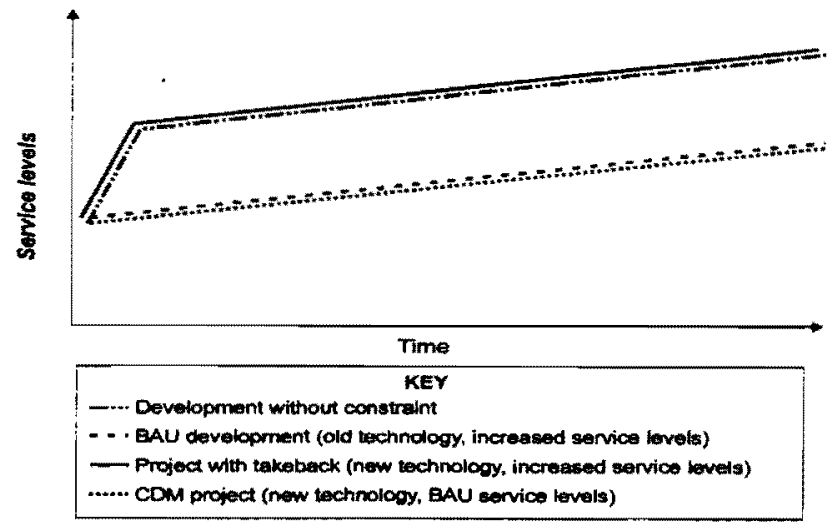


The changes in service levels in the four cases are summarised in Figure 3. Clearly the CDM project with take-back and unconstrained development have higher levels of service than BAU development or the CDM project without take-back.

What are the implications for calculating CERs? Referring back to Figure 1 we argue that only Area A should be counted for CERs for the investors, showing the difference before and after the CDM project taking into account the increased consumption. This gives the CDM investors a return on the real emissions reductions, but not the part taken back by the household in response to its energy savings (Area B) which is additional emissions attributable to increased service and hence part of the sustainable development benefit. We argue that the difference between the development that would have happened without constraint and $\mathrm{BAU}$ development (Area $\mathrm{C}$ ) should be credited to the host countries or entities within the host country (e.g. the project owners). The host country might choose to re-invest its CERs into the project, in order to promote projects that have a high sustainable development benefit. A practical difficulty that needs to be resolved is how the level of "satisfied demand" or demand without constraint is determined.

\subsection{Fuel switching for rural lighting}

Roy (2000: 434-436) gives an example of the take-back effect in relation to rural lighting. The project example cited dates back to surveys undertaken in 1995 and explains fuel switching from subsidised kerosene fuelled lamps to subsidised solar lanterns for lighting.

Before the introduction of the solar lanterns, kerosene was used for lighting on a household average rate of consumption of 2.5 litres per week. When the lanterns were introduced, kerosene consumption for lighting was expected to be replaced. What did happen was that lighting quality improved (from 10 to 380 lumens) and the level of activity increased from 2 to 4 hours per night to 6 hours per night. Part of the quality and quantity of unsatisfied demand are hereby met.

Initial situation, before the CDM: 2.5 litres/week.

After the CDM: 0 litres/week (same level of activity as BAU).

The calculated saving therefore was expected to be 2.5 litres per week (except in seasons when there was insufficient sunshine). However, the actual saving was ranged between 1.25 and 0.5 litres per month. The 50 per cent to 80 per cent kerosene take back effect was attributed to saved kerosene being used meet the increased demand for lighting. Not included in this take-back is the saved kerosene that was used to meet previously suppressed cooking services (only 
partly met with biomass) and to on-sell kerosene to other members of the community. Roy (2000: 435$)$ suggests that if the increase in activity is totalled, the combined take back could be in the region of 200 per cent.

After the CDM (2): 1.25 to 2 litres/week (with lighting take-back).

After the CDM (3): 5 litres/week (with lighting and cooking take-back).

The business-as-usual (BAU) scenario assumes a beginning at the point of the project intervention and proceed at an increased growth of kerosene consumption (consistent with the expected growth rate in commercial energy consumption expected in India) at $5.5 \%$ per annum. This increase in both lighting and cooking services is the result of, amongst others factors, increasing population and income. In relative terms, the income constraint is relaxed somewhat over time, but demand is still not saturated.

Business-as-usual growth: 2.5 litres/week * $(1+0.055) /$ year.

Development without constraint: levels of activity for service are at a saturated/satisfied level using BAU technologies immediately. The example could be explained diagrammatically in Figure 3.

As in the previous example, the areas A and B in Figure 3 show the difference before and after the project. Since energy consumption (and hence emissions) grow over time in each case, the sum of the two areas represents litres of kerosene which, multiplied by an emissions factor provides an indication of the total emissions reductions. However, area $B$ represents emissions avoided by moving to a cleaner development path from business as usual and is the sustainable development benefit. Area $\mathrm{C}$ provides the amount of kerosene that would be required to meet a kerosene fuelled lighting demand at a satisfied lighting service benchmark of so many lumens being related to $\mathrm{X}$ litres per week, less the measured CDM project CERs in lighting with the lighting takeback. The cooking takeback can, like area $\mathrm{B}$, be considered a sustainable development benefit, that may have to be counted when leakage is considered in compiling a project development document. Though in this case, the leakage is closely linked to the introduction of a second subsidides fuel/energy service which has increased the affordability of kerosene fuelled energy services in part replaced by $P V$. 


\section{Figure 3 Consumption of kerosene for lighting for rural lighting}

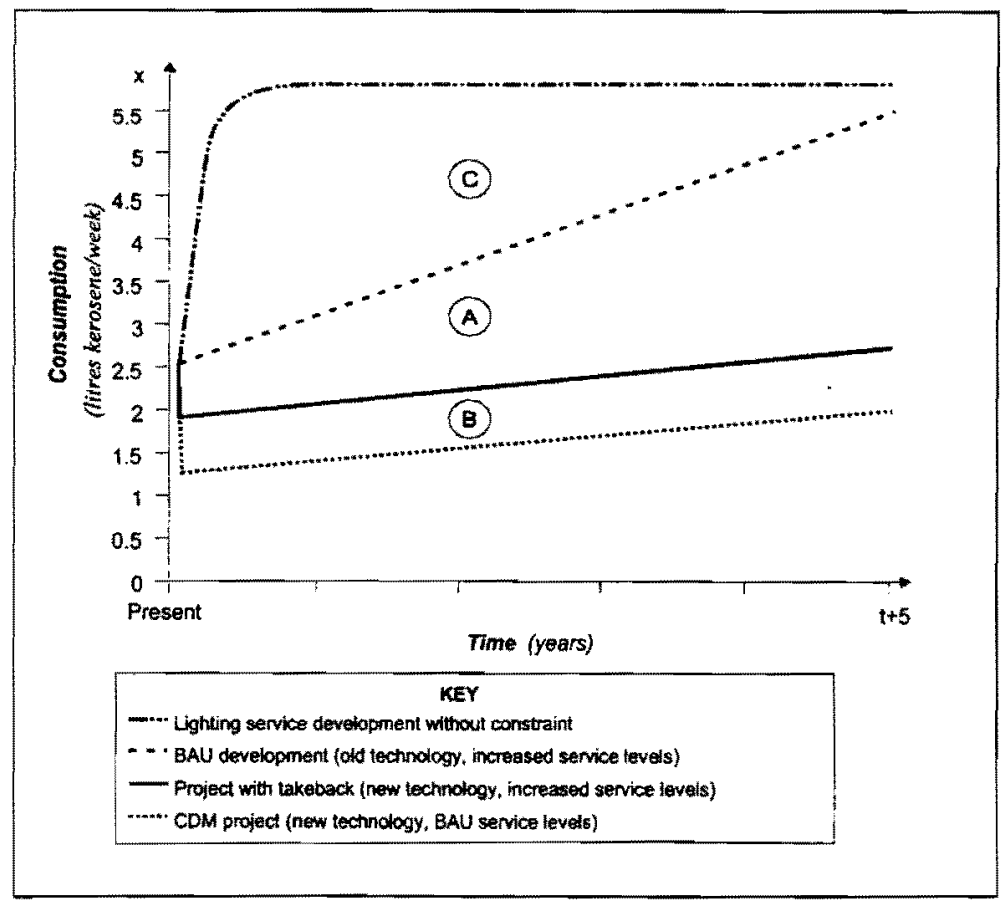

The total Certified Emissions Reductions (CERs) is area A plus area C multiplied by the emissions factor of kerosene - less any emissions associated with the solar lantern. This amount includes the benefits of the efficiency improvements (A) and credits the suppressed lighting demand (C). The CERs represented by Area $A$ accrue to the investor. The host country might bank credits from $\mathrm{C}$ against possible future commitments, project owners might wish to sell them on the market or they could be re-invested in the project to make it more attractive to investors. Area $B$ does not receive CERs, but results in a development benefit of increased service levels.

The increase in the level of activity, is equivalent to a formal contribution to energy poverty alleviation. Roy suggests that the pre-efficiency improvement situation is like "a constrained optimum case" where constraint comes from lack of infrastructure (electricity or kerosene) or low-income. She concludes "in such a situation change in the technical efficiency improvement acted like relaxation of constraint." A surprising conclusion is that fuel demand was higher after rather than before the solar lantern introduction. This seems to indicate that the 
introduction of cleaner energy technologies can contribute to addressing energy poverty.

The current energy consumption in India indicates that Indians use 3 per cent of what US citizens use on a per capita basis (Roy, 2000). The high growth rate of 6 per cent and the less than unitary elasticity of commercial energy use (0.92) implies a high potential level for growth in emissions at a rate of 5.5 per cent. India is not alone in this predicament. Speculation is that "even with aggressive policies to address energy efficiency, developing countries" energy demand is likely to grow five- to ten-fold over the next 30 to 40 year, resulting in a threefold increase in world energy demand" (Roy, 2000). These scenarios add up to a compelling case to design and implement climate-related policy interventions that address this possibility before it becomes a reality. Addressing suppressed demand is now one such measure of increasing levels of service while coincidentally decreasing emissions intensity of the new clean technologies.

\subsection{Starting from minimal service levels: SHS example}

Where there is currently minimal (close to zero ${ }^{4}$ ) levels of service, the approach to suppressed demand baselines must be modified. An example of Solar Home Systems (SHS) provided by Lazarus et al (1999) illustrates this point. The results can differ depending whether a benchmark or project-specific baseline is used.

The CDM project introduces SHS by reducing initial costs to rural households. As a results, the households run televisions or small refrigerators, services they did not have before the CDM project and were unlikely to acquire in the absence of the CDM project. A similar logic would apply in any off-grid electricity project, or other project categories were there is currently very low or zero levels of service.

Before the CDM (project-specific approach): $0 \mathrm{kWh} / \mathrm{mth}$, therefore 0 emissions.

The CDM project, therefore, results in activity (i.e., use of energy services) that is additional to the reference situation without the CDM project. Assume that the SHS program results in ancillary emissions of $0.1 \mathrm{~kg} \mathrm{CO} / \mathrm{kWh}$, and the benchmark baseline for rural electricity is set nationally at $1.0 \mathrm{~kg} \mathrm{CO} / \mathrm{kWh}^{5}$, roughly equivalent to the emissions of small diesel generators (Lazarus $e t$ al., 1999: Box 2.1).

Before the CDM (benchmark): $1.0 \mathrm{~kg} \mathrm{CO} / \mathrm{kWh}$. 
Whether the project generates any CERs depends on whether a benchmark or project-specific baseline is used. Compared to the national benchmark, emissions are $0.9 \mathrm{~kg} \mathrm{CO} 2$ lower for every $\mathrm{kWh}$ generated. Effectively this does not take into account actual current activity levels.

After the CDM (benchmark): $(1.0 \mathrm{~kg} \mathrm{CO} / \mathrm{kWh}-0.1 \mathrm{~kg} \mathrm{CO} / \mathrm{kWh}) *$ activity of SHS.

The project-specific approach considers both activity and intensity. The SHS project would not be credited, since total emissions increase in comparison to the baseline scenario of no energy services.

After the CDM (project-specific): project emissions -0 emissions $=$ no credit.

Lazarus et al. (1999) argue that the project might still be credited under the project-specific approach because it is impossible to predict the counterfactual situation with any certainty (random error) or because it is difficult to refute the claim that the activity would have occurred anyway (gaming and systematic error) (Lazarus et al., 1999).

\section{A BASELINE METHODOLOGY IN SITUATIONS OF SUPPRESSED DEMAND}

How should CDM project baselines deal with conditions of suppressed demand? As a general approach, we have argued that in conditions of suppressed demand, one cannot assume that activity levels will remain constant. Increases in activity levels are allowed in terms of the CDM rules. Credit should be given where a project assists the developing country in moving directly to a cleaner development path, rather than first adopting old ("dirtier") technologies. Similar to the fast-track provisions in the CDM rules, this requires focusing on the greater public benefit and accepting some non-additional emission reductions.

This paper has illustrated the approach with selected examples. The examples illustrate three more general groups of projects - those with take-back effect, fuel-switching and minimal service levels. Where some of the emissions reductions are counter-acted by the take-back effect, we suggest that:

- The increased service levels of the same energy service using the business as usual technology should form part of the baseline calculations; 
- Increases in service levels of other energy services should be counted as sustainable development benefits to the host country, but not included in baseline calculations. They are likely to be relatively small;

- CERs accruing to the investor should be calculated as the difference between BAU development and the CDM project with the take-back effect related to the same energy service; and

- Host country entities should have discretion over credits for the difference between development without constraint and BAU development under suppressed demand.

Where fuel switching occurs, take-back would certainly be considered for the same service. Whether take-back used for increases in other services should be counted as sustainable development benefits or included in the baseline, requires further study.

In situations where there are minimal (close to zero) levels of $z$ 'service, sustainable development must mean increasing service levels. The recommendation from the case study is that credit should be given against a benchmark, rather than taking a project-specific approach.

\section{CONCLUSION}

It is apparent that wherever there are opportunities for relaxing activity/demand constraints there are possibilities for CDM interventions using a suppressed demand baseline methodology. Examples of interventions will therefore exist wherever there is poverty and/or lack of infrastructure. The methodology outlined here could be applied equally to other sectors, including some grid expansion CDM projects where currently services are going unmet or met at low levels of service and other situations of suppressed demand. Household, transport, small and medium enterprises, small-scale agriculture (water pumping, tilling etc.), greenfields projects providing a new service and energy services are all likely to qualify precisely because the level of activity is constrained and will grow faster once efficient technologies are introduced.

The methodology suggested in this paper raises questions that will need to be addressed in practical application to real CDM projects. This short paper could not attempt to address all questions that arise. A specific area requiring further investigation is what host country credits might be used for. Another is what level of energy services might be considered 'satisfied' and whether this might be an intemational, regional or local standard. 
The UNFCCC and the CDM both have sustainable development among their objectives. Sustainable development will require increased levels of service in many instances, particularly where there is suppressed demand. This needs to be taken into account in baseline calculations. This approach will need to be presented to the CDM Executive Board to make an in-principle decision, so that it can be applied to projects that will raise the concern.

By far the most important outcome of such an approach to baseline methodologies is that it establishes a formal modality for using flexible mechanisms to attract investments that contribute to poverty alleviation and infrastructural underdevelopment. If this is achievable, and it looks as if the text would allow for this, it would strengthen the sustainable development contribution of the CDM.

\section{ENDNOTES}

1 Different terms are used in various aspects of the climate change debate suppressed demand, avoided emissions, unsatisfied demand and growth baselines. Each has its own associations; we use suppressed demand in this paper and explain the concept here

2 Both terms are used in the literature; we use take-back effect in this article.

3 An alternative CDM project would be to reduce the price of CFLs to a level where this barrier is removed.

4 It is tempting to talk of zero service levels, but even in very poor households, there is some kind of lighting service, even if the levels are appallingly low. Public transport might be an example where one can more accurately speak of zero service levels.

5 We follow the factor used by Lazarus et al. (1999) for simplicity. A weighted average for planned facilities for electricity generation in SA showed a similar value of $0.83 \mathrm{kgCO}_{2} / \mathrm{kWh}$ (Winkler et al. 2000)

\section{REFERENCES}

1 BAUMERT, K. (1999) "Understanding Additionality", (ed.) Promoting Development while Limiting Greenhouse Gas Emissions: Trends \& Baselines, New York, World Resources Institute \& United Nations Development Programme: 135-145.

2 BERNOW, S., KARTHA, S., LAZARUS, M. \& PAGE, T. (2000) Cleaner Generation, Free Riders and Environmental Integrity: Clean Development Mechanism and the Power Sector, Boston, Tellus Institute. 
3 CLARK, A. (1997) "Economic Analysis of Eskom's. Energy-Efficient Lighting Programme for Low-Income Households", Energy \& Development Research Centre, University of Cape Town.

4 ESKOM (2000) Efficient Lighting Initiative. Menlo Park, Eskom.

5 GREENING, L.A., GREENE, D.L. \& DIFIGLIO, C. (2000) "Energy Efficiency and Consumption - the Rebound Effect - a Survey", Energy Policy 28 (6-7): 389-401.

6 LAZARUS, M., KARTHA, S. \& BERNOW, S. (2000) Key Issues in Benchmark Baselines for the CDM: Aggregation, Stringency, Cohorts and Updating, Boston, Tellus Institute.

7 LAZARUS, M., KARTHA, S. \& BERNOW, S. (2001) "Project Baselines and Boundaries for Project-Based GHG Emission Reduction Trading", Boston, Tellus Institute.

8 LAZARUS, M., KARTHA, S., RUTH, M., BERNOW, S. \& DUNMIRE, C. (1999) Evaluation of Benchmarking as an Approach for Establishing Clean Development Mechanism Baselines, Boston, Tellus Institute and Stratus Consulting.

9 MEYERS, S. (1999) Additionality of Emissions Reductions from Clean Development Mechanism Projects: Issues and Options for Project-Level Assessment, LBNL-43704, Berkeley, Lawrence Berkeley National Laboratory.

10 MEYERS, S. (2000) Determining Baselines and Additionality for the Clean Development Mechanism: Are Simplified Methods Viable? Berkeley, Lawrence Berkeley National Laboratory.

11 MICHAELOWA, A. \& DUTSCHKE, M. (1999) "Economic and Political Aspects of Baselines in the CDM Context. WRI and UNDP" (ed.) Promoting Development while Limiting Greenhouse Gas Emissions: Trends \& baselines. New York, World Resources Institute \& United Nations Development Programme: 115-134.

12 NICHOLSON, W. (1995) Microeconomic theory: Basic principles and extensions. Fort Worth, Dryden Press.

13 OECD \& IEA (Organisation for Economic Co-operation and Development, International Energy Agency) (2000) Emission baselines. Paris.

14 ROY, J. (2000) The rebound effect: some empirical evidence from India. Energy Policy 28 (6-7): 433-438.

15 SATHAYE, J. (2001) Baselines, Data Needs, and Other Methodological Challenges: Technical Considerations. Sustainable climate change projects: training on host country program development, Cape Town, US Department of Energy, US Agency for International Development \& US Environmental Protection Agency. 3-5 April.

16 UNFCCC (1992) United Nations Framework Convention on Climate Change. New York, United Nations, http://www.unfccc.de/resource/conv/ conv.htm. 
17 UNFCCC (1997) Kyoto Protocol to the United Nations Framework Convention on Climate Change, Bonn, UNFCCC Secretariat, www.unfecc.de.

18 UNFCCC (United Nations Framework Convention on Climate Change) (2001) Report of the Conference of the Parties on its Seventh Session", Held at Marrakesh from 29 October-10 November 2001, FCCC/CP/2001/13, Marrakesh, COP-7, www.unfccc.int.

19 WINKLER, H., SPALDING-FECHER, R, AFRANE-OKESE, Y. \& DAVIDSON, O. (2000) "Potential Multi-Project Baselines in the Power Sector in South Africa", Presented at the Sixth Conference of the Parties to the UN Framework Convention on Climate Change, Cape Town, Energy \& Development Research Centre and Lawrence Berkeley National Laboratory. 